International Journal of Engineering \& Technology, $7(3.3)(2018) 82-86$
International Journal of Engineering \& Technology
SPC
Website: www.sciencepubco.com/index.php/IJET
Research paper

\title{
A Protocol for the Effective Utilization of Energy in Wireless Sensor Network
}

\author{
Santosh Anand ${ }^{1 *}$, Pillai Atulya Radhakrishna ${ }^{2}$ \\ Department of Computer Science \\ Amrita School of Arts and Sciences, Mysuru \\ Amrita Vishwa Vidyapeetham, India \\ *Corresponding author E-mail:santoshanand.jha@gmail.com
}

\begin{abstract}
In current technology Adhoc network is most commonly used in communication network. Major parts of the Adhoc network devices are based on the sensor nodes. Sensor devices operated on the battery so energy is vital constraint. Effective Utilization of the energy of the sensor nodes and sensor networks to the full extent is highly impracticable. Optimization of the network root and the load balancing in coverage helps to maximize the lifetime of the sensor network considerably. The research carry out on sensor network, deployment model is proposed which essentially finds the optimized roots with the help of neighbour discovery algorithm which find the neighbour and non neighbour nodes and analysis the load of the network based on Packet Retrieval in RREQ, throughput, average energy consumption, packet delivery ratio and packet drop.
\end{abstract}

Keywords: $A O D V ; D S D V ; D S R ; R R E Q ; W S N$.

\section{Introduction}

Wireless sensor networks (WSN), are like wireless ad hoc networks as it rely on wireless accessibility and unrestrained arrangement of networks with the purpose that sensor data may be transported wireless. WSN is also dust networks, alluding to minute sensors as low as dust. Smart dust is a U C Berkeley venture supported by DARPA. Dust Networks Inc. is one of the early organizations that transport component of wireless sensor network. WSNs are involved inappropriate self-decision sensors to physical or ecological conditions, for instance, temperature, weight, noise, etc and to supportively move that data from the system to essential zones. In the current technology networks are bi-directional, moreover engaged in manage of sensor development. The military applications propelled the headway of wireless sensor networks. Today such networks are used for various mechanical and buyer applications, for instance, modern process checking and control, machine prosperity observing, and so on.The WSN is working within number of sensor nodes, where each node is related with one (or group of) sensors. Each sensor organize hub, which has frequently a group of segments. The expenditure of sensor nodes is due to many factors. Size and rate of the sensor nodes get contrasting objectives on resources, for instance, essentialness, speed, memory, and exchanges of data.
WSN's topology can move to the multi-hop wireless from an essential star system.

\section{Literature survey}

"Qianqian Yang et.al [1][2015] In this work author proposed a probabilistic sensing technique, this technique is more realistic to characterize the region of sensing of the sensor nodes. It compares the threshold value from multiple user's and combines the sensing probability and if it is threshold then it covers a point. Author further designed the "Full Area Coverage Optimization" is used to choose a part of sensors nodes and which dynamically give probability area coverage to extend the sensor network lifecycle as much as possible.

JieTian et.al [2][2015] In this work an algorithm is proposed for routing in WSN and for joint scheduling. This algorithm will improve connectivity of network, reduces the power consumption and having the scheduling and routing algorithm which can switch off each disjoint node sequentially to achieve for balancing energy consumption while packet transmission.

R. Kacimi et.al [3][2013] In this work author proposed a strategy for consumption the energy of the nodes and maximizes the lifespan of the network by balancing the traffic. First, formailze the problem of maximizing the life of sensor network and then optimizes the load balancing solution.

S. K. A. Imon et.al [4][2013] RaSMaLai efficient algorithm is implemented to achieve maximum lifetime of the WSN. called 
randomized switching for maximizing the life of WSN. They have further designed a D-RaSMaLai which is distributed algorithm and the results achieved shows that the algorithm proposed is satisfactory than the existing methods according to the network lifetime.

A.Jarry et.al [5][2011] The proposed methodology used by the author an algorithm for distributed algorithm through online which balances the energy in optimized network structures and there are stable in thee network. Which is proved that the life time of the network is improved.

P. Chatterjee et.al [6][2011]This system is based on non-uniform randomly node is distributed and developed an algorithm for data gathering which is distributed and distribute the traffic uniformly to increase the life span of the network.

X. Zhang et.al[7][2010]In this, author worked on the improvement of lifetime of randomly distributed node in WSN. Author proposed static distribution technique which can gather the data based on neighbour node to achieve the minimum energy consumption.

A. Chamam et.al[8][2009] The energy hole problem in WSN are studied in non-uniform node distributed strategy. Author proposed non-uniform node distribution technique to balance consumption of energy in the network.

X. Wu et.al [9][2008 In this system, sensor nodes send data from multiple sensors to a sensor network, and each sensor node generates CBR ("Constant Bit Rate"). This sensor network is used in many applications, such as tracking and collecting data. According to this observation, the nodes are located around receivers that need to broadcast traffic to other nodes.

J.C.Dagher et.al [10][2007]In this work, author proposed a novel theory that maximizes the life of unicast multi-hop WSN. An iterative algorithm is an optimal solution. This algorithm finds the Pareto optimal (PO) solution.

P. Casari et.al [11][2006]The life of the sensor nodes reply on the number of time the collection of data is performed without any nodes exhausting power. In this paper, a specific task is considered to provide information of each node to the sink node.

A. Jarry et.al [12][2006] An algorithm proposed by the author is based on distribution and adaptive data propagation to balance the energy between the sensors nodes in the network and proposed hybrid routing technique which allows every nodes to send a information to its immediate neighbours, which depends on the potential function of its remaining energy.

J. Li et.al [13][2005]In this system, a new mathematical tool for optimizing routing and topology designed in WSN. Author introduced a formula based on the vector field which is used to represent the communication in the network and perform routing based on the vector field. The density of data volume passing through the location reply on the size of the vector field and defines the total cost of the communication in the network.

A. Giridhar et.al [14][2005] In this work, the authors propose an algorithm to decide whether to transmit data to the destination (receiver) or send the data directly to the receiver. This randomly chosen balanced (cheap) one-hop transmission is directly transmitted to the receiver, which is more expensive, but the "bypass" sensor is close to the receiver.

Manishankar.S et.al [15] [2017] In this work, the efficiency of the network is measured through a certain parameters such as time delay, jitter, end to end delay and packet loss.
Mukhopadhyay et.al [16][2016] The work proposed by author is vehicular Adhoc Networks are efficiently supported with LTE due to its mobility rate which is extraordinary and it is unique QoS parameters for LTE."

\section{Proposed methodology}

Studies carried on the existing literature surveys gave a detailed idea on problems that are associated with network performance. The improvement that has to be made in the network is the deployment model and enhancement of associated routing protocol. The research over here proposes an optimized path routing algorithm and an efficient traffic analysis and control.

The network environment contains sensor nodes which are employed in a FlatGrid pattern. The data received from communication nodes is tracked and details of initial connections or path is collected. Collected data will be forward to path optimizer which in turn uses neighbour discovery techniques and it suggests an optimal path. Performance tracker is included for collecting details such as energy, time delay and packet drop.

The primitive algorithm used in path optimization is modified Dijkstra algorithm which works with single source shortest path.

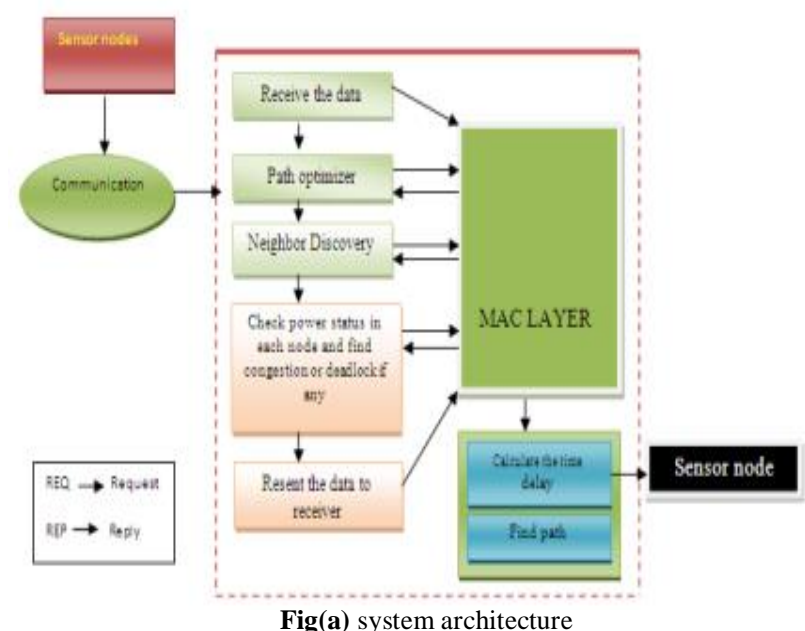

\subsection{Path Optimizer Algorithm}

Proposed work is implemented in the forms of algorithm. Steps:

1. Choose a source node $s$ from group of nodes $n$.

2. Generate a traffic e between $s$ and remaining $n-1$ nodes.

3. Calculate the number of hop between $s$ and other nodes

4. Classify the n-1 into two list 'nl' and 'nnl' based on hop count.

5. Calculate the path value ' $\mathrm{P}$ ' for the neighbour list.

6. Apply modified Dijkstra's algorithm on ' $\mathrm{nl}$ '.

dis $=\operatorname{sqrt}(((\operatorname{des} \mathrm{X}-\mathrm{nl}[\mathrm{h}, \mathrm{n}]) *(\operatorname{des} \mathrm{X}-\mathrm{nl}[\mathrm{h}, \mathrm{n}]))+((\operatorname{des} \mathrm{Y}-$

$\mathrm{nl}[\mathrm{h}, \mathrm{n}+1]) *(\operatorname{des} Y-\mathrm{nl}[\mathrm{h}, \mathrm{n}+1])))$

Recursively execute the algorithm for other nodes.

7. Set a threshold value for distance and compare it with value obtained from Dijkstra.

8. If obtained value less than threshold distance then add it to optimal path list.

9. If path does not exist in the list update the path list.

10. Else omit the founded path.

11. Calculate the on Packet Retrieval in RREQ, throughput, average energy consumption, packet delivery ratio and packet drop.

12. In case of new node communication search among the path list, the path with Packet Retrieval in RREQ, throughput, average energy consumption, packet delivery ratio and packet drop.

Table (1) Parameter of path optimizer

\begin{tabular}{|l|l|}
\hline Symbol & Definition \\
\hline S & Source \\
\hline N & Number of nodes \\
\hline
\end{tabular}




\begin{tabular}{|l|l|}
\hline Nl & Neighbour list \\
\hline Nnl & Non neighbour list \\
\hline $\mathrm{H}$ & Number of hop \\
\hline $\mathrm{P}$ & Path \\
\hline $\mathrm{S}$ & Source \\
\hline
\end{tabular}

Categorization of nodes is done with the help of the threshold value set by applying the neighbour discovery technique. Distance between nodes can be calculated with the method:

Dist $\left.=\sqrt{ }([(\mathrm{x} 2-\mathrm{x} 1))]^{\wedge} 2+\llbracket(\mathrm{y} 2-\mathrm{y} 1) \rrbracket^{\wedge} 2\right)$

The neighbour discovery technique check whether it is less than the threshold or greater than the threshold. If it is less than the threshold than it is a neighbour node and if it is greater than the threshold than it is non neighbour node.

\subsection{Communication Protocol}

AODV (Ad Hoc on Demand distance Vector) protocol is used as the routing protocol in this work. It merges some important properties of the Dynamic Source Routing (DSR) and DestinationSequenced Distance-Vector Routing (DSDV). It is used to improve the lifetime of the nodes because it creates the routes only when there are any packets to travels from source to destination. It helps to reduce maintains of each and every nodes in route. It also helps in minimizing the count of nodes in the route between the source and sink node.

AODV protocol provides routing table for unicast routes and also for the multicast routes. It handles route disclosure with Route Request (RREQ) messages. RREQ message is communicated to neighbour hubs. Grouping numbers are utilized to ensure circle opportunity RREQ messages cause avoided hubs to allot route table sections for turnaround route. Hubs transmitting a RREP message make routing table sections for forward route.

It is one of the leading responsive protocols and has the advantages of DSR and DSDV protocol. DSDV shows all the paths available to all destinations with minimum count of nodes to the destination AODV inherits the succession numbers of DSDV and minimizes the flooding of course information by creating courses on-request, and improves the routing scalability and efficiency of DSR, which carries the source course in the information parcel. In AODV protocol, it finds a course to the destination, the source broadcasts a course ask for parcel (RREQ). Its neighbours relay the RREQ to their neighbours until the RREQ achieves the destination or an intermediate hub that has new course information. Then the destination or this intermediate hub sends a course answer bundle (RREP) to the source hub along the way from which the first duplicate of the RREQ is received. AODV utilizes grouping numbers to determine whether course information is sufficiently new and to guarantee that the courses are sans circle. It can acknowledge very rapidly to the topological adjustments that affect the active routes due to its adaptability to highly dynamic networks. It has very less delay for connections and detection of the recent route to the destination. Routes do not include the packet header.

If a node wants to send some information to any destination, the protocol checks if there is any current route to the destination in the routing table, then it transmit packets through the route. If it is not available then initiates the path from the source sensor node to receiver sensor node. From source node the path is created by creating the RREQ (Route Request packet). The packets send from the source node will contain many fields which help to reach the destination. When RREQ reaches to the node, the node will create a reverse route to the source node. By using this reverse path a node can transmit a RREP (Route Reply packet) to the source sensor node. The RREQ reaches the destination when the fields' matches with the address in the RREQ packets then the destination sends the RREP packets to source. If the address does not matches then it increment the hop count in RREQ and broadcast to any neighbour node. At the end the RREQ will reach the destination.

\section{Result and discussion}

This research work is based on the mobile Adhoc network. The simulation of the network is carried out with the help of the NS2 simulator. The network was designed with the Flat Grid topology and the network consists of $\mathrm{n}$ number of nodes which shown in fig(b). The Adhoc network was made up with the help of WIFI channels and basic routing protocols are used.

The parameters of initial network are:

Table (2) network parameter

\begin{tabular}{|l|l|}
\hline Type & Value \\
\hline Mactype & Mac/802_11 \\
\hline Queue & droptrail/ priqueue \\
\hline Propagation type & Two way ground \\
\hline Channel type & Wireless channel \\
\hline idle power & 0.5 \\
\hline Rxpower & 1.5 \\
\hline Txpower & 2.0 \\
\hline initial energy & 100 \\
\hline
\end{tabular}

Nodes are created according to the table 2 parameters and nodes were initialized with mobility modules. Each and every nodes are assigned with $\mathrm{x}$ position $\mathrm{y}$ position and $\mathrm{z}$ position value in order for calculating the distance and in turn to measure the network performance. Each node initial distances were marked and colours were assigned in order to track the changes while deploying the network.

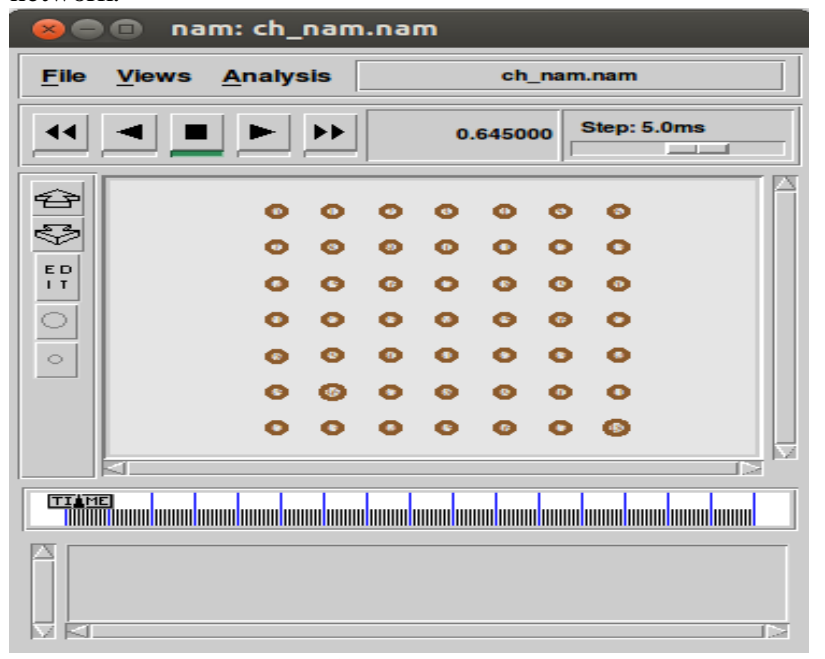

Fig (b) Creation of nodes

Procedure for initializing the mobility in the network was done with the randomized regular expression which is shown in fig(c). A sink node is created and utilized in order to track the packet loss and monitor the network. A constant bit rate traffic is generated in the network and packet flow in UDP is stable. The broadcast communications are used with RREQ and RREP message. Generated data path from source sensor node to destination sensor node were notated with the help of a specific colour which can be observed from fig (d).

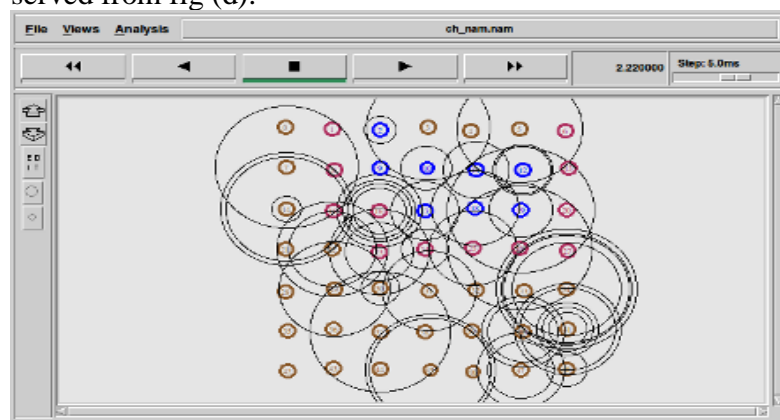

C.

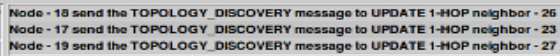

Fig (c) Mobility of the nodes. 
After the initial broadcast, the distance value is obtained through communication. It gives an opportunity to find the neighbour and non-neighbouring nodes and the transmission distance between various communicating nodes.

In this work, the proposed algorithm for path optimization of the network is brought into the picture. The algorithm initially run to checks the neighbours which are in the reachable of one hop and prints the position as well as the direct broadcast distance. Further algorithm takes the non-neighbouring nodes in consideration and the distance calculation procedure is created then positions and distances are put into a list.

The vital part of the algorithm lies in shortest path calculation and distance optimization considering the intermediate adjacent nodes occurring between neighbour node and non-neighbour node. Minimum threshold set distance and the recursive calculation of indirect distance of each iteration is combined and shortest path is calculated. The colour is set of the specific path obtained through path optimized algorithm and if the path does not exist the operation is terminated and successive node is consisted.

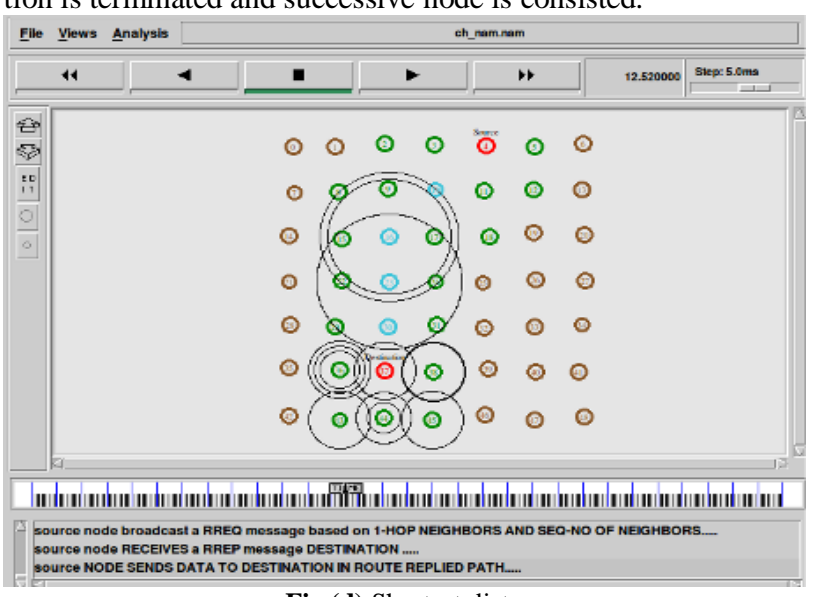

Fig (d) Shortest distance.

All the values obtained throughout the network operation are recorded into the specified $\operatorname{tr}(\operatorname{trace})$ file which is taken as the input for the comparison graph of all three protocols implementation. The different algorithm were tested such as AODV, DSR and then comparison is done on the basis of the throughput, packet retrieval, packet delivery ratio, Energy Consumption and packet drop.

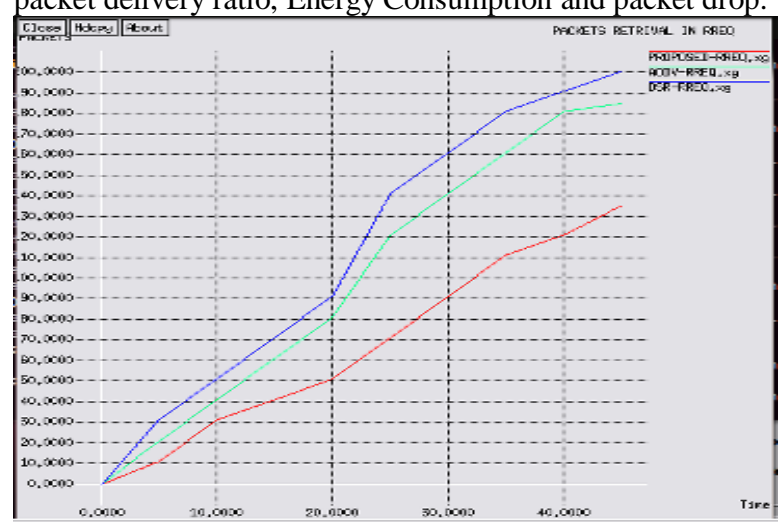

Fig (e) Packet Retrieval in RREQ

The graph depicted in fig(e) represents the average packets retrieval in RREQ. The graph shows that the proposed model RREQ as the lowest packet lost and better retrieval compare to AODV and DSR. The red colour in the graph is the proposed algorithm packet retrieval. The green colour in the graph is the AODV RREQ and the blue colour in the graph is the DSR RREQ.

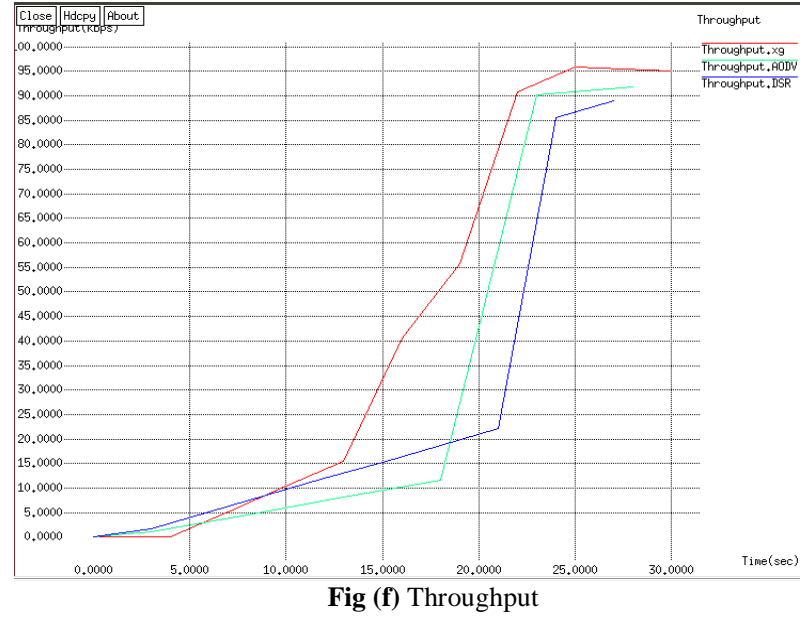

The graph in the fig (f) represents the overall throughput of the algorithms that are executed in order to make an efficient network and also for the optimization. It is observed that the proposed system has better throughput efficiency than AODV and DSR. In the above graph red colour represents the proposed system throughput and the green and blue colour represents AODV and DSR respectively.

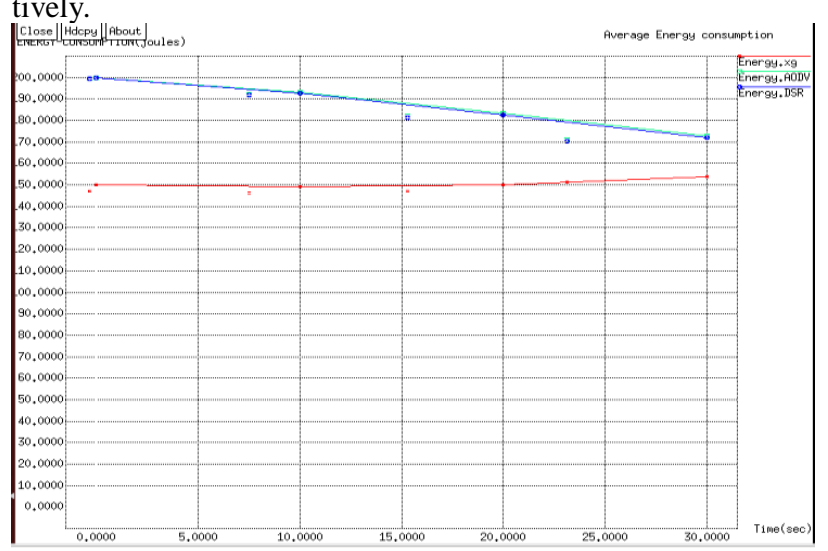

Fig (g) Average Energy Consumption.

The graph depicted in fig $(\mathrm{g})$ represents the average energy consumption of the algorithms. AODV and DSR have similar energy utilization and compare to them the proposed algorithm have lower and constant energy utilization. The red colour represents the proposed algorithm energy consumption and the green and blue colour is the AODV and DSR algorithm energy consumptions respectively

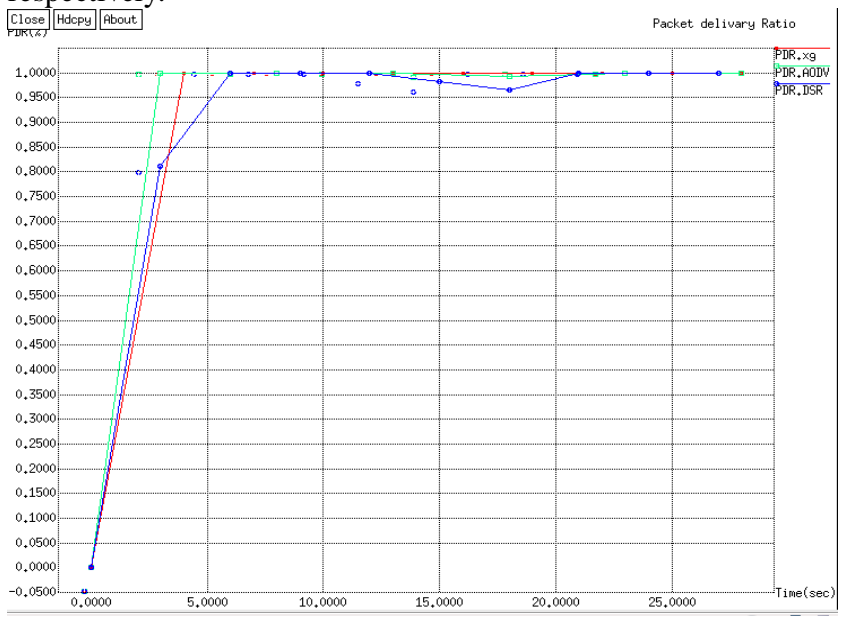

Fig (h) Packet delivery ratio.

The graph depicted in the fig $(\mathrm{h})$ represents the packet delivery ratio(PDR) of the algorithms. The PDR seam to reach an optimal level in all the three algorithms but in the initial stages the delivery 
ratio is better for the proposed algorithm and it does not lead to scarcity.

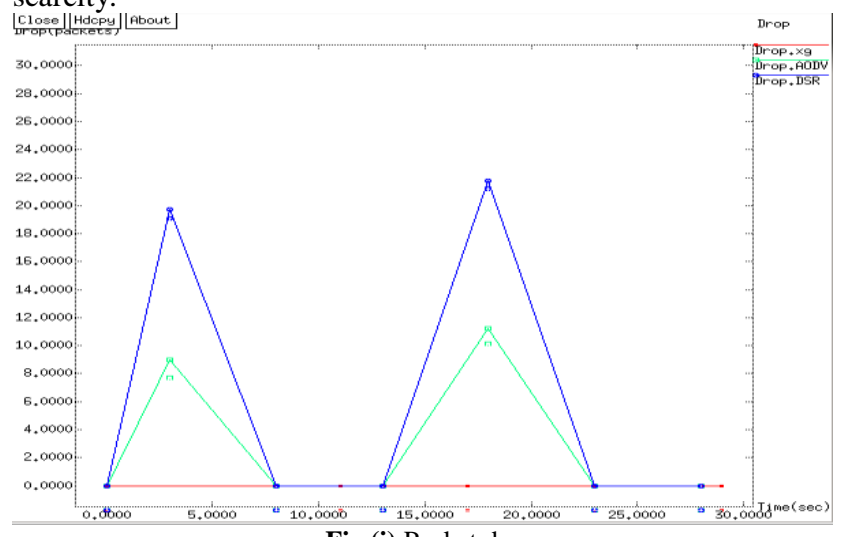

Fig (i) Packet drop

The graph shown in fig (i) represents the packet drops in various algorithms. Packet drop is minimal level and does not seem to increase in the proposed system compare to AODV and DSR where there are sudden increases with respect to packet drop.

\section{Conclusion}

The research carried out covers details about sensor network deployment model and various ways to optimize the network performance. The optimal roots are discovered and performance parameters are evaluated. The sensor network deployment is done using NS2 and comparison of existing routing algorithm with the proposed algorithm is carried out by considering the different performance criteria. The system can be enhanced further by adding energy consumption and sensor motion behaviour.

\section{References}

[1] Qianqian Yang, Shibo He, Junkun Li, Jiming Chen and Youxian Sun, "Energy-Efficient Probabilistic Area Coverage in Wireless Sensor Networks", IEEE Transactions on Vehicular Technology, vol. 64, no.1, pp. 367-377, January 2015

[2] JieTian, Xiaoyuan Liang, Tan Yan, Mahesh Kumar Somashekar,Guiling Wang, and Cesar Bandera, "A Novel Set Division Algorithm for Joint Scheduling and Routing in Wireless Sensor Networks", Springer Wireless Networks, vol. 21, Issue 5, pp. 1443-1455, July 2015.

[3] R. Kacimi, R. Dhaou and A.-L.Beylot, "Load balancing techniques for lifetime maximizing in wireless sensor networks", Ad Hoc Networks Journal, vol. 11, no. 8, pp. 2172-2186, 2013.

[4] S. K. A. Imon, A. Khan, M. D. Francesco and S. K. Das, "RaSMaLai: A Randomized Switching Algorithm for Maximizing Lifetime in Treebased Wireless Sensor Networks", Proc. IEEE INFOCOM, pp. 2913-2921, 2013.

[5] A. Jarry, P. Leone, S. E. Nikoletseas and J. D. P. Rolim, "Optimal data gathering paths and energy-balance mechanisms in wireless networks", Ad Hoc Networks Journal, vol. 9, no.6, pp.1036-1048, 2011.

[6] P. Chatterjee and N. Das, "Distributed Data Gathering with GradedNode Distribution in Sensor Networks to Maximize Lifetime", Proc. of the 25th International Conference on AdvancedInformationNetworking and Applications (AINA), IEEE, pp. 435-442 Singapore, March 2011.

[7] X. Zhang, Z. D. Wu, "A Non-Uniform Node Deployment Approachfor Event Detection Sensor Networks", IEEE 29th International Performance Computing and Communications Conference (IPCCC) 2010, pp. 42 - 49.

[8] A. Chamam, "On the Planning of Wireless Sensor Networks: Energy-Efficient Clustering under the Joint Routing and Coverage Constraint", IEEE Transactions on Mobile Computing, vol. 8, no. 8, pp.1077-1086, August 2009.

[9] X. Wu, G. Chen and S. K. Das, "Avoiding Energy Holes in Wireless Sensor Networks with Nonuniform Node Distribution", IEEE Transactions on parallel and distributed systems, vol. 19, no. 5,pp.710-720, May 2008
[10] J.C. Dagher, M.W. Marcellin and M.A. Neifeld, "A theory for maximizing the lifetime of sensor networks", IEEE Transactions on Communications,vol. 55, no. 2, pp.323-332, 2007.

[11] P. Casari, M. Nati, C. Petrioli and M. Zorzi, "ALBA: An Adaptive Load-Balanced Algorithm For Geographic Forwarding in Wireless Sensor Networks", IEEE MILCOM 2006, pp. 1-9.

[12] A. Jarry, P. Leone, O. Powell and J. Rolim, "An optimal data propagation algorithm for maximizing the lifespan of sensor networks, Proc.IEEE International Conference on Distributed Computing in Sensor Systems", pp 405-421, June 2006, San Francisco, USA.

[13] J. Li and P. Mohapatra, "An Analytical Model for the Energy Hole Problem in Many-to-One Sensor Networks", Proc. 62nd IEEE Vehicular Technology Conference, pp. 2721-2725, 2005.

[14] A. Giridhar and P.R. Kumar, "Maximizing the functional lifetime of sensor networks", Proc. 4th international symposium on Information processing in sensor networks, pp. 5-12, 2005, Los Angeles, California.

[15] S. Manishankar, C. R. Srinithi and D. Joseph, "Comprehensive study of wireless networks QOS parameters and comparing their performance based on real time scenario," 2017 International Conference on Innovations in Information, Embedded and Communication Systems (ICIIECS), Coimbatore, India, 2017, pp. 1-6.

[16] Mukhopadhyay, Adwitiya, S. Raghunath, and M. Kruti."Feasibility and performance evaluation of VANET techniques to enhance realtime emergency healthcare services." Advances in Computing, Communications andInformatics (ICACCI), 2016 International Conference on. IEEE, 2016. 